1 Computing flooding of crossroads with obstacles using a 2D numerical model

3 PIERRE-HENRI BAZIN, PhD, Irstea, UR HHLY, 5 rue de la Doua BP 32138, 69616 Villeurbanne

4 Cedex, France

$5 \quad$ Email : pierrehenri.bazin@gmail.com.

6 EMMANUEL MIGNOT, Assistant Professor, Laboratoire de Mécanique des Fluides et d'Acoustique

7 (LMFA), CNRS-Université de Lyon, Ecole Centrale de Lyon/Université Lyon 1/INSA de Lyon, 20

8 Avenue A. Einstein, 69621 Villeurbanne, France

9 Email : emmanuel.mignot@insa-lyon.fr (author for correspondence)

10 ANDRE PAQUIER (IAHR Member), Engineer, Irstea, UR HHLY, 5 rue de la Doua BP32138, 69616

11 Villeurbanne Cedex, France

12 Email:andre.paquier@irstea.fr.

13

14 Running Head: Computing flooding of crossroads with obstacles

15

16 


\section{Computing flooding of crossroads with obstacles using a 2D numerical model}

\section{ABSTRACT}

Typical urban flood flow features are usually computed using two-dimensional numerical models. How such modelling can be implemented in dense urban areas with obstacles is investigated. A strategy for representing the effect of urban obstacles in various flow conditions is defined. The comparison between the available laboratory measurements and the model results show that if the water depth is high enough and the flow remains subcritical, two-dimensional modelling with constant eddy viscosity provides the effect of the obstacles on the flow distribution accurately, even with a coarse mesh; moreover, an over-simplified representation of the sidewalks averaging the street cross section elevations seems sufficient. Oppositely, if the water depth is low and/or the flow becomes supercritical, the description of the flow is not relevant enough and it generates errors in the flow distribution at the crossroads.

Keywords: Flood modelling; flow-structure interaction; junction flow; two-dimensional models; urban flood

\section{Introduction}

According to the European Environment Agency report (EEA, 2010), floods have caused 1126 deaths in the period 1998-2009. Besides, floods remain the most costly natural hazard, with cumulated damages evaluated to EUR 52 billion in the same period. Actually, most of the human losses and economic damages occur in the urban areas that concentrate a large part of the stakes: population, economic activities and industries, historical centres, road networks... because cities are often located in flood-prone areas such as river floodplains and coastal areas.

When considering a dense urban area, the flood can be assumed to occur mainly in the street network so that surface flow models can be restrained to this network (e.g. Leandro, Chen, Djordjevic, \& Savic, 2009; Mignot, Paquier, \& Haider, 2006; Mignot, Paquier, \& Ishigaki, 2006). The standard approach to simulate these events relies on a depth averaged two-dimensional model (2D) solving the full shallow water equations which provides the global flood extent and the spatial distribution of the maximum water levels, along with detailed time series of local flow depths and velocities. The ability of standard 2D models to represent the global surface flow pattern during urban floods has been assessed by comparing numerical simulations and laboratory experiments for idealized and wellcontrolled urban configurations corresponding to flooded urban crossroads (El Kadi Abderrezzak, Lewicki, Paquier, Riviere, \& Travin 2011; Ghostine et al., 2010; Mignot, Paquier, \& Riviere, 2008) or schematic flooded urban areas (Mignot, Paquier, \& Ishigaki, 2006; Soares-Frazão, \& Zech 2008; Van Emelen et al., 2012). 
Nevertheless, obstacles of dimensions much smaller than the buildings such as bus-stops, trees or parked cars, that are common street furniture, are never included in the flood simulations even if these obstacles may strongly modify the flow pattern and thus the risk distribution in the city. Moreover, given the increasing computing capacities and spatial data acquisition methods from existing GIS databases or Lidar land survey, such introduction of urban details is not a limiting process anymore.

Mignot et al. (2013) recently measured and computed (using a 3D code solving the unsteady Reynolds-averaged Navier-Stokes equations with a Spalart-Allmaras model for turbulence closure) the impact of obstacles located in a crossroad on flow distribution to the downstream streets. The chosen geometry was an idealized subcritical 3-branch dividing flow. The main results in terms of impact on the flow distribution are listed below:

- The impact of the obstacle on the discharge distribution is strongly dependent on its location with regards to the crossroad. Indeed, obstacles located within the upstream channel increase the streamwise flow velocity and thus tend to reduce the lateral and increase the downstream discharges. Oppositely obstacles located within the downstream channel tend to block off the flow in this channel and to reduce the corresponding discharge while increasing the lateral discharge. Finally for obstacles located within the branch channel, their impact depends on the side of the channel in which they are introduced.

- This impact is a direct consequence of the modifications when introducing the obstacle of the: (i) streamwise and centrifugal flow acceleration, (ii) width of the recirculation zone and (iii) wake downstream the obstacle.

- The impact of an obstacle appears to increase as the upstream Froude number increases while the modification of the normalized water depth hardly affects the results and the impact of the initial flow distribution depends on the obstacle location.

Given the potential impacts of a single obstacle located in the vicinity of the bifurcation (modification of the flow distribution up to $12 \%$ ), the present paper aims at verifying the capacities of operational 2D numerical models to reproduce these impacts.

2D modelling of subcritical dividing flows in a three branch bifurcation without obstacle was carried out by Shettar and Murthy (1996) and Khan, Cadavid, and Wang (2000). Both models proved an excellent ability to compute the discharge distribution. In particular, Shettar and Murthy (1996) performed an extensive validation of the numerical model, using vertically-averaged velocity fields, water surface profiles at the intersection, as well as more global flow characteristics such as the size of the branch separation zone and the energy loss in the junction.

On the other hand, simulations of flows around obstacles in the literature mainly concern flows around bridge piers or groynes and are preferably computed using 3D numerical models (as in Mignot et al., 2013), but 2D models are also used along with adequate turbulence closure models. 
Yulistiyanto, Zech, and Graf (1998) used a 2D model to simulate the flow around an emerging cylinder using a specific treatment of the dispersion stresses due to vertical velocity profiles and their simulations appear to fairly predict the velocities and water depths around the cylinder, without any calibration. Jiang, Yang, and Liang (2009) performed 2D modelling of the flow past a vertical plate, and proved that an eddy viscosity model computed via the friction velocity can achieve reasonable prediction of the velocity field. Stansby (2006) performed simulations of the flow past a conical island with a 2D model, including a horizontal mixing-length turbulence eddy viscosity model and the ability of the model was found to depend on the wake type, with discrepancies increasing when predicting the occurrence and length of stable wakes.

Nevertheless, operational 2D numerical models used for urban flood modelling rarely consider turbulence effects, or use simple turbulence models because accurate modelling of turbulence would require computational efforts that are not affordable for large-scale flood studies. Moreover, the modellers often neglect the presence of obstacles and simply do not consider them in the geometry/meshing process. Then the present paper aims at modelling the impact in the crossroad of obstacles from Mignot et al. (2013) and sidewalks from Bazin (2013) using a 2D operational numerical model with a very simple turbulence model, as used when modelling the urban flood events, in order to reveal that the consideration of such obstacle could improve the usual urban flood calculation. This model is thus much simpler than the 3D model previously used by Mignot et al. (2013) to compute these flows but this latter model could not be applied to urban flooding calculations. The objective is here twofold: 1) to identify for which obstacle location and flow configuration a 2D operational model is able to fairly estimate the large-scale effects (mostly the effect of the discharge distribution to the downstream branches) of an impervious obstacle or a sidewalk in the vicinity of a 3-branch open-channel bifurcation and 2) to identify which meshing strategy (method for including obstacles and selected mesh dimensions) is required to achieve this fair estimate. The first section presents experimental data used herein along with the 2D numerical model and its application in cases where no obstacle is included, the second section presents the calculation of flows in bifurcations with each obstacle configuration and then with the sidewalks.

\section{Experimental data and numerical model}

\subsection{Experimental data}

The experimental data are derived from measurements performed by Mignot et al. (2013) and Bazin (2013). The chosen geometry was an idealized subcritical 3-branch dividing flow with one inlet named "upstream channel" with subscript " $u$ " and two outlets, one aligned with the inlet named "downstream channel" with subscript "d" and the second forming a $90^{\circ}$ angle, named "branch channel" with subscript "b". The experiments included 14 flow configurations listed in Table 1 with varying dimensionless parameters $\mathrm{F}_{\mathrm{u} 0}($ series $\mathrm{S} 1), R_{q 0}(\mathrm{~S} 2)$ and $h_{u 0} / b(\mathrm{~S} 3)$, where $\mathrm{F}$ is the Froude number, $R_{q}=$ 
$121 Q_{b} / Q_{u}$ is the flow distribution with $Q$ the discharge, $b$ is the channel width, $h$ is the water depth and

122 with the 0 subscript referring to the case without obstacle. For each of these 14 flows, the discharge

123 distribution $R_{\mathrm{q}}$ was measured without obstacle (denoted as $R_{q 0}$ ) and after adding one obstacle at each

124 of the 7 locations sketched in Fig. 1, replacing the subscript 0 (without obstacle) by the subscripts 1 to

1257 corresponding to the obstacle location number. The obstacles are square shape impervious and

126 emerging blocks of base dimensions $5 \mathrm{~cm}$ x $5 \mathrm{~cm}$. Note that Mignot et al. (2013) also measured

127 configurations with two obstacles but these configurations are not considered herein. However, for the

128 same 14 flows, sidewalks (of dimensions $6 \mathrm{~cm} \times 2 \mathrm{~cm}$, Fig. 1b) were also added (without obstacles) by

129 Bazin (2013) and this additional configuration is referred to by the subscript 10 as sketched in Fig.1.

130 In the end, a total of 14 flows times 9 cases were measured (1 without obstacle, 7 with one obstacle

131 and 1 with sidewalk), that is 126 flow configurations. Additionally, the $2 \mathrm{D}$ horizontal velocity field

132 was measured at one selected elevation $(z=3 \mathrm{~cm}, z / h=0.71)$ for flow 6 (Table 1) without obstacle, with

133 all obstacles, and finally with the sidewalks. All these experimental configurations can be considered

134 to be representative of a crossroad of narrow streets in a city at scale 1/25 (Mignot et al., 2013).

\subsection{Equations and numerical model}

136 The Rubar20 code solves the 2D shallow water equations including the continuity equation (Eq. 1) and

137 the conservation of momentum along orthogonal axes $x$ and $y$ (Eqs 2 and 3):

$$
\begin{aligned}
& \frac{\partial(h u)}{\partial t}+\frac{\partial\left(h u^{2}\right)}{\partial x}+\frac{\partial(h u v)}{\partial y}+g h \frac{\partial h}{\partial x}=-g h \frac{\partial z_{b}}{\partial x}-g \frac{u \sqrt{u^{2}+v^{2}}}{K_{s}{ }^{2}{ }^{1 / 3}}+K\left[\frac{\partial}{\partial x}\left(h \frac{\partial u}{\partial x}\right)+\frac{\partial}{\partial y}\left(h \frac{\partial u}{\partial y}\right)\right] \\
& \frac{\partial(h v)}{\partial t}+\frac{\partial\left(h v^{2}\right)}{\partial y}+\frac{\partial(h u v)}{\partial x}+g h \frac{\partial h}{\partial y}=-g h \frac{\partial z_{b}}{\partial y}-g \frac{v \sqrt{u^{2}+v^{2}}}{K_{s}{ }^{2} h^{1 / 3}}+K\left[\frac{\partial}{\partial x}\left(h \frac{\partial v}{\partial x}\right)+\frac{\partial}{\partial y}\left(h \frac{\partial v}{\partial y}\right)\right]
\end{aligned}
$$

141 where $t$ is the time, $h$ is the water depth, $u$ and $v$ are the depth averaged velocities along respectively $x$

142 and $y$ axis, $g$ is the gravity acceleration, $Z_{b}$ is the bottom elevation, $K_{\mathrm{s}}$ is the Strickler coefficient with

$143 K_{\mathrm{s}}=1 / n, n$ being the Manning roughness coefficient, and $K$ is the eddy viscosity coefficient. The eddy

144 viscosity coefficient $K$ represents effects of diffusion, depth-averaging of the velocities as well as

145 turbulent stresses; it does not form an elaborate turbulence model but it offers a way to calibrate

146 simulations in the case where turbulence effects have to be considered. The simplest formulation for $K$

147 assumes a constant value in time and space. Use of such simple eddy viscosity model can lead to

148 acceptable results once $K$ is calibrated, as shown for flows including strong two-dimensional patterns

149 such as separations zones (Bravo \& Holly, 1996; Papanicolaou, Elhakeem, \& Wardman, 2011).

150 Depending on the experimental flow configurations, the flow regime is here either

151 hydraulically smooth or in the transition toward fully rough flow. Therefore, as for Mignot, Paquier, 
and Rivière (2008), the following explicit approximation of the Colebrook-White formula given by Yen (2002) is considered for computing the friction factor $f$ :

$$
f=\frac{1}{4}\left[-\log _{10}\left(\frac{k_{S}}{12 R_{h}}+\frac{6.79}{\mathrm{R}^{0.9}}\right)\right]^{-2}
$$

where $\mathrm{R}$ is the local Reynolds number calculated as $4 h \sqrt{u^{2}+v^{2}} / v$ with $v$ the water kinematic viscosity, $R_{h}$ is the hydraulic radius, taken here as the local water depth $h$ and $k_{s}=0.1 \mathrm{~mm}$ the channel roughness height. The computed friction factor is then transformed in an equivalent Strickler coefficient for Eqs 2 and 3 using:

$$
K_{S}=\sqrt{\frac{8 g}{h^{1 / 3 f}}}
$$

The usual range of equivalent Strickler coefficients is $85-105 \mathrm{~m}^{1 / 3} \mathrm{~s}^{-1}$. Moreover, for cells located along solid boundaries (including the obstacles), wall friction is added, considering it equal to half the bottom friction for the same water depth (for simplicity and because the averaged water pressure along the wall should be half the water pressure at the bottom). Finally, the obstacles are represented as impervious cells in which no flow is calculated. The sidewalks are represented in the topography increasing the bottom elevation of all the points of the sidewalks by their elevation, i.e. by $0.02 \mathrm{~m}$.

mesh is made of quadrilaterals and triangles using an unstructured grid so that the mesh can be adapted to any complex obstacle shape. The code solves the above equations using an explicit secondorder finite-volume scheme, adapted from MUSCL approach. The numerical scheme can run with a fixed time step, or with an adaptive time step respecting a Courant number below 1 so that the scheme remains stable. Originally developed for simulations of dam-break waves, the code is robust for all the simulations of shallow flows. Treatment of the drying/wetting processes is by setting null water depths whenever the computed ones are lower than a threshold $(0.001 \mathrm{~mm}$ for computations described in the present paper). The mass conservation is achieved with typical errors less than $0.01 \%$ of the total mass. Code validation against experimental and field data includes simulations of dividing supercritical and transcritical flows (Mignot, Paquier, \& Rivière, 2008; El Kadi Abderrezzak et al., 2011), floods in dense urban areas (Mignot, Paquier, \& Ishigaki, 2006) and dam-break type flows around obstacles (El Kadi Abderrezzak, Paquier, \& Mignot, 2009). The main discrepancies observed in previous comparisons with laboratory measurements concern the exact prediction of the location and size of the hydraulic jumps.

In the present study, the reference mesh consists of a square grid with a resolution of $m=3.5$ to $5 \mathrm{~cm}$ in the junction, leading to 7 cells across the channels. This mesh size is indeed representative of a model used for simulating urban flood events. This mesh permits to capture the time-averaged flow around each obstacle, but may not represent finer phenomenon such as vortex shedding (note that 
Lloyd \& Stansby (1997) and Yulistiyanto et al. (1998) use around 30 cells across their obstacles). A refined mesh ( $m=0.5 \mathrm{~cm}$ in the junction and $2 \mathrm{~cm}$ in the channels) is used for verifying the mesh influence on the results. The inlet flow discharge $Q_{u 0}$ experimentally measured in the pumping loop is imposed on the total width of the upstream channel inlet, with a uniform velocity distribution across the boundary. The downstream boundary conditions imposed at the outlet sections of the downstream and lateral branches are the experimental weir equations, i.e. the measured stage-discharge relationships $Q_{b}=\mathrm{f}\left(C_{b}, h_{b}\right)$ and $Q_{d}=\mathrm{f}\left(C_{d}, h_{d}\right)$ with $C$ the weir crest height and $h$ the water depth measured two channel widths upstream from the weir.

\subsection{Numerical validation: modelling flows without obstacles}

195 First of all, the measured channel roughness height equals $0.1 \mathrm{~mm}$ and it was verified (not shown here) that a variation of this parameter within the range of uncertainty ( 0.05 to $0.2 \mathrm{~mm}$ ) does not impact the calculations. Now, the parameters subject to calibration and their acceptable ranges are the mesh size (from $0.5 \mathrm{~cm}$ to $3.5-5 \mathrm{~cm}$ ) and the eddy viscosity (from 0 to $10^{-3} \mathrm{~m}^{2} \mathrm{~s}^{-1}$ ). In order to validate the numerical model ability to simulate the discharge distribution and predict the general flow patterns before introducing the obstacles and to identify the best option for the two calibration parameters, seven parameter runs detailed in Table 2 are used to compute the 14 flows without obstacles (subscript 0 ), considering the two mesh sizes and four values of eddy viscosity coefficient $K$. First, the measured water depths are compared to calculated ones resulting in an average difference of about $0.1 \mathrm{~mm}$ and a mean quadratic difference of $0.6 \mathrm{~mm}$ (not shown here), which can be considered as small compared to an average water depth of about 40 to $50 \mathrm{~mm}$ and the accuracy of the water depth measurements. Then, the capacity to predict the discharge distribution is assessed calculating the average $\delta$ and root mean square deviation $\sigma$ of the relative error of the lateral discharge $Q_{b 0}^{*}$ with:

$$
\begin{gathered}
Q_{b 0}^{*}=\frac{Q_{b 0, S I M}-Q_{b 0, M E S}}{Q_{b 0, M E S}} \\
\delta\left(Q_{b 0}^{*}\right)=\frac{1}{14} \sum_{k=1}^{14} Q_{b 0}^{*} \\
\sigma\left(Q_{b 0}^{*}\right)=\sqrt{\frac{\sum_{k=1}^{14} Q_{b 0}^{*}}{14}}
\end{gathered}
$$

where $Q_{b 0, S I M}$ and $Q_{b 0, M E S}$ are respectively the simulated and measured values of the branch channel

212 flow discharge obtained without obstacles. These indicators included in columns 4-5 of Table 2

213 confirm the capacities of the numerical model to estimate the discharge distribution, with typical

214 errors about $2 \%$, and a bias generally negative $\left(\delta\left(Q_{b 0}^{*}\right)<0\right)$. These results do not promote a specific 215 value for the eddy viscosity K (runs A-B-C or E1-E2-D-E3) and reveal that the mesh refinement (runs 216 E1-E2-D-E3) only slightly improves the calculation adequacy with regards to the use of a coarser 217 mesh (runs A-B-C). The optimum seems to be run B as it considers a coarser mesh (of same size as 218 the obstacle dimension, that is the minimum size that could be used for simulations with obstacles) 
and a minimum $\sigma\left(Q^{*}{ }_{b 0}\right)$. Nevertheless, the optimum run without obstacle may not be the optimum run when considering the obstacles. For instance, the computation of the wake created by the obstacle may require a finer mesh than that of run $\mathrm{B}$. As a consequence, four runs are kept for future analysis with obstacles: the three cases with the coarse mesh with different eddy viscosity values (A, B and C) and a fourth one with the finer mesh (D) in order to verify whether a mesh refinement improves the calculations of the flow with obstacles.

Figure 2 compares the horizontal measured and simulated velocity fields for flow number 6 (Table 1). Note that experimental velocities are measured at the elevation $z=3 \mathrm{~cm}(z / h \sim 0.71)$, slightly above the mid elevation, which are likely to be representative of the depth-averaged velocity, whereas the computed velocities are depth-averaged velocities. Within this comparison framework, the data from the 4 parameter runs appear to be in fair agreement with experimental measurements both quantitatively and qualitatively except for the transverse extension (along $x$ ) of the recirculation cell in the downstream branch (Fig. 2b). The coarse mesh (runs A, B and C) leads to a coarse representation of the velocity field, but the global flow pattern remains well predicted without any clear advantage while varying the eddy viscosity coefficient.

The recirculation area located along the upstream wall of the branch channel is a main flow structure in a subcritical dividing flow (Mignot et al., 2014). From the experimental and numerical data, the contour of the recirculation zone is computed, assuming it can be taken as the "zerodischarge area". Figure 3 shows the contour of this recirculation area for flow 6 (Table 1), for measured data and computed runs $\mathrm{A}$ and $\mathrm{C}\left(K=0\right.$ and $\left.K=10^{-3} \mathrm{~m}^{2} \mathrm{~s}^{-1}\right)$. For run A, the recirculation area almost reaches the downstream weir of the branch channel, whereas its length (along $y$ axis) strongly decreases for run C. Oppositely, the maximum width of the recirculation appears to be similar for both $K$ values.

To conclude, the numerical model appears to predict the experimental flows without obstacle with enough accuracy to allow the introduction of obstacles and sidewalks in the next section.

\section{Modelling of flows with obstacles or sidewalks}

\subsection{Introduction of obstacles}

246 Here below, the 14 flows are simulated with the 7 obstacles configurations from Fig. 1a and the 4

247 parameter runs $\mathrm{A}, \mathrm{B}, \mathrm{C}$ and $\mathrm{D}$, representing a total of $14 \times 7 \times 4=392$ calculations. First, each simulation 248 is assessed calculating the simulated and measured discharge distribution modification due to the 249 obstacle:

$$
\Delta R_{q i, S I M}=R_{q i, S I M}-R_{q 0, S I M}
$$

$$
\Delta R_{q i, M E S}=R_{q i, M E S}-R_{q 0, M E S}
$$


and then comparing the simulated and measured cases:

$$
\Delta R_{q i}=\Delta R_{q i, S I M}-\Delta R_{q i, M E S}
$$

with $\mathrm{i}=1 \ldots 7$ the obstacle number (Fig.1). Then, for each parameter set the average $\delta$ and root mean square deviation $\sigma$ of these errors are computed over the 7 obstacle configurations and the 14 flow configurations, in a similar way as in Eqs. 7 and 8 and are indicated in columns 4-5 of Table 3. Moreover, the accuracy of the model to compute the discharge distribution with obstacle is included in columns 6-7 of Table 3 with:

$$
\delta\left(Q_{b 1-7}^{*}\right)=\frac{1}{14} \sum_{k=1}^{14}\left(\frac{1}{7} \sum_{i=1}^{7} Q_{b i}^{*}\right)
$$

$$
\sigma\left(Q_{b 1-7}^{*}\right)=\sqrt{\frac{\sum_{k=1}^{14} \sum_{i=1}^{7}\left(Q_{b i}^{*}{ }^{2}\right)}{14 \times 7}}
$$

Table 3 reveals an overall fair ability to predict the discharge distribution when obstacles are included with a typical average error lower than $3 \%$ and a root mean square error of about $3 \%$. It appears that:

- using the fine (D) and coarse (B) meshes results in a similar agreement with the measurements.

- the bias $\delta$ decreases as the eddy viscosity coefficient decreases (C-B-A) which means that the calculated branch discharge is reduced

- the higher values of the eddy viscosity coefficient (runs B-C) seem to be the best options although the differences using the three values remain weak.

- the error of the simulation with obstacles is of the same order as the error for the flows without obstacles provided in Table 2

Scatter plots comparing measured and simulated evolutions of the discharge distribution are shown on Fig.4 for runs B (coarse mesh) and D (fine mesh). A linear regression using a least-square method is carried out and the slope $s$ is included, showing that the calculations fairly estimate the discharge distribution for most of the 98 calculations using each mesh.

Still using runs B and D, Fig.5 details for each obstacle and each series from Table 1 the measured and simulated modifications of the discharge distribution as each obstacle is introduced. Figure 5 confirms that for both parameter runs (corresponding to both mesh refinements), most obstacle configurations (each line) and most flows (each column), the discharge distribution is well estimated by the numerical model; i.e. computed and measured symbols are in agreement.

Discrepancies mainly occur for: which the flow without obstacle includes a significant part of supercritical regime in the 
junction. The simulation of this partially supercritical flow with obstacles 1, 2, 6 and 7 is less accurate than for other flows with lower Froude numbers. This limit is related to a slight uncertainty in the prediction of the actual hydraulic jump location, as already noticed by Mignot, Paquier, and Rivière (2008).

- the upstream obstacle 2 for which $\left|\Delta R_{q 2}\right|$ is overestimated when computed with the fine mesh (run D) and underestimated when computed with the coarser one (run B).

In order to gain information on the computational errors when introducing obstacle 2, Fig.6 shows the horizontal velocity fields measured (at $z=3 \mathrm{~cm}$ ) and computed (depth-averaged velocity field) for flow 6 with the 4 parameter runs (to be compared with the measured flow without obstacle in Fig.2). It appears that when using the coarse mesh (runs A to C), the size of the wake with small velocity downstream of the obstacle is strongly overestimated, which limits the flow capacity to rotate into the branch channel. Thus, there is an increased effect of obstacle 2, hence an increase of $\left|\Delta R_{q 2}\right|$ but the coarse mesh also smoothens the flow around obstacle 2, so that finally the effect of the obstacle on the discharge distribution is rather fairly estimated for this flow 6, as shown on Fig.5. Oppositely, the use of the fine mesh (D) on Fig.6a leads to a shorter wake and thus to a higher deviation of the flow towards the branch and thus a slightly better estimate of $\Delta R_{q 2}$ shown on Fig.5. Moreover, Fig. 6b reveals that both meshes lead to a fair estimation of the velocity field within the intersection $(x<0.3 \mathrm{~m}$ and $y<0.3 \mathrm{~m}$ ) but not to a precise extension of the recirculation zone, as already observed without obstacle (Fig. 2b). For this obstacle 2, additional simulations were carried out for all flows with the fine mesh using friction velocity-dependent eddy viscosity formulations. None of these additional runs achieved a better agreement with the measured discharge distribution (not shown here for the sake of scarcity).

\subsection{Introduction of sidewalks}

As the sidewalks containing vertical edges are submerged, the meshing challenge lies in an adequate representation of their topography. Cells slope in 2D models is limited by their dimensions for practical reasons (computation times), so an adaptation of the mesh is required. The mesh considered in the present section is made of regular $2 \mathrm{~cm}$ width square elements everywhere except on the sidewalks edges, where finer elements of $0.5 \mathrm{~cm}$ or $1 \mathrm{~cm}$ are used (Fig.7). Using this mesh, two different topographical representations are used (Fig. 7):

- the reference topography (Ref) similar to the experimental geometry. constant and equals the average elevation of the channel cross section, i.e. $2 \times(6 \mathrm{~cm} \times 2 \mathrm{~cm}) /(30 \mathrm{~cm})=0.8 \mathrm{~cm}$. This representation is the simpler representation of the topographical change induced by the sidewalks. 
4 parameter runs are defined in Table 4, using both topographical representations and two eddy viscosity coefficient values $K$. As for obstacles in Table 3, the average error $\delta$ and root mean square error $\sigma$ of the predicted discharge distribution and evolution of the discharge distribution when including the sidewalks are included in Table 4, with the subscript 10 referring to the sidewalks. It appears that:

- the model is able to predict the discharge distribution when sidewalks are included with a typical average error lower than $3 \%$ and a root mean square error of about $5 \%$.

- the simplified topographical representation $A v g$ gives similar results as the reference configuration, without main deterioration of the results.

- slightly better simulation results are obtained using a higher eddy viscosity coefficient $K=10^{-3}$ $\mathrm{m}^{2} \mathrm{~s}^{-1}$.

Figure 8 shows the measured and horizontal simulated velocity fields for flow 6 , for parameter runs 1 and 2 from Table 4 carried out with the Ref topography (to be compared with the measured flow without sidewalk in Fig.2). The acceleration of the flow in the upstream channel central area is well simulated but this acceleration does not extend within the intersection in the numerical model, unlike in the measurements (Fig. 8b). Moreover, the lateral extension of the recirculation and adjacent acceleration zones in the branch channel are fairly (Fig. 8c) but not perfectly (Fig. 8b) estimated. Moreover, increasing the eddy viscosity (run 2) hardly affects the velocity distribution across the channel width, and only slightly changes the branch channel recirculation shape.

Measured and simulated modifications of the discharge distribution due to the sidewalks are shown for each of the 14 flows on Fig.9 in the same way as for Fig.5. Results with the 4 runs appear to be close to each other, and stand well within the range of experimental uncertainties, except:

- for flow 2 that has a large upstream channel Froude number. The discontinuity (sudden $\Delta R_{q}$ decrease as $F_{u 0}$ increases) observed in the experiments for $F_{u 0} \sim 0.6-0.8$ is rather occurring for $F_{\mathrm{u} 0} \sim 0.4-0.6$ in the numerical simulations. Figure 10 shows that this discontinuity is due to the occurrence of an oblique hydraulic jump attached to the upstream corner of the junction (Mignot, Riviere, Perkins, \& Paquier, 2008), which strongly affects the flow. The location of the hydraulic jump is not perfectly estimated by the numerical model and leads to the strong error in the discharge distribution for flow 2; but to a fair estimation for flows 1 and 3 (Fig. 9). This result confirms the conclusion raised by Mignot, Paquier, and Rivière (2008) that a slight error in the hydraulic jump location can (but not necessarily) lead to strong errors in the discharge distribution.

- for flow 11 that is the shallowest flow configuration $\left(h_{u 0} / b=0.08\right)$ for which the water depth $\left(h_{u 0}=2.4 \mathrm{~cm}\right)$ hardly exceeds the elevation of the sidewalk $(z=2 \mathrm{~cm})$, so that the flow conditions do not fit the shallow water equations hypothesis. 


\section{Conclusions}

354 Numerical simulations of experimental bifurcation flows have been carried out with the Rubar20 code that solves the two-dimensional shallow water equations. Comparison of simulation results with experimental measurements allowed to assess the code ability to model nine series (one without obstacle, seven with one single obstacle and one with sidewalks) of 14 subcritical dividing flows with varying Froude number, discharge distribution and water depths. The computation of the discharge distribution for the flows without obstacles/sidewalks was achieved with a fair accuracy (error typically less than $2 \%$, Table 2), without specific calibration of the numerical model although better results are obtained using a small positive value of the eddy viscosity $K$ (typically $5 \times 10^{-4}$ to $10^{-3} \mathrm{~m}^{2} / \mathrm{s}$ for the cases considered herein). Therefore, the prediction of the discharge distribution for subcritical dividing flows with the shallow water equations (code Rubar20) appears to be more accurate than for supercritical (Mignot, Paquier, \& Rivière, 2008) or transcritical flows (El Kadi Abderrezzak et al., 2011).

The simulations of the flows with obstacle proved the capacities of the code to fairly predict the effects of one obstacle on the flow (deflections, contractions and accelerations) and the consequences on the discharge distribution to the downstream channels. It appeared that a relatively coarse mesh ( 3.5 to $5 \mathrm{~cm}$ cells, i.e. of same size as the typical size of the obstacle) is sufficient to fairly compute the large-scale effects (mostly the flow distribution to the downstream branches) of the obstacle. Deeper analysis shows that errors mainly arise i) when hydraulic jumps take place in the crossroad and/or ii) when the obstacle is introduced in the upstream channel (obstacles 1 and 2), the wake of which strongly impacts the flow in the area where it is deflected towards the branch channel. Improving this calculation may require the use of a finer mesh and a more elaborate turbulence model, but both aspects would be too costly to be included when simulating urban floods in large urban areas.

The authors wish to warn any potential user of the present model that, regarding the test functions $\delta$ and $\sigma$ indicated in Tables 2 and 3, the model appears to be substantially insensitive to the calibrated parameters within their range of variations considered herein. Nevertheless, we showed that these parameters do affect specific details of the flow patterns such as the recirculation zone (an increasing $K$ value tends to reduce its length as shown in Fig. 3) or the wake downstream the obstacle (a refined mesh tends to reduce the wake streamwise extension in Fig. 6a and leads to a better estimate of the discharge distribution in Fig. 5).

Modelling of flows with sidewalks was performed with two topographical models and both fairly predict the impact of the sidewalks on the discharge distribution. The calculations with the simplified geometry show that the simulation of the average flow acceleration in a channel section is sufficient to predict the impact of the sidewalks. The main discrepancies appear to be related to 1) the occurrence of supercritical flows in the crossroad or 2) a very limited water depth over the sidewalk. This suggests that the effect of the sidewalks is mainly related to the upstream flow acceleration, and 
that there is no significant impact on the mechanism of flow division (except when the flow in the

390 junction becomes supercritical).

391 Finally, the potential change in flow distribution due to obstacles of typical dimension equal to

$3921 / 6$ of the street in a subcritical flow with aspect ratios ranging between 0.08 and 0.22 can exceed

$39310 \%$. These results are likely to be generalized to a large set of urban cases because the laboratory

394 experiments are scaled to a specific crossroad in a city and are using physically based shallow water

395 equations. On the other hand, the literature (Mignot, Paquier, \& Haider, 2006; Paquier \& Bazin, 2014)

396 showed that the uncertainty associated to other parameters of the urban flood calculation such as the

397 hydrographs, neglecting the sewage network, some open areas within blocks, or the rain falling on the

398 computed domain may lead to comparable levels of uncertainty. Because the inclusion of obstacles

399 does not require a more detailed mesh, modellers could easily explicitly integrate such obstacles into

400 their urban flood models, which would improve the calculation of the flow distribution and the

401 representation of the local flow patterns. Nevertheless, the error made when neglecting obstacles of

402 smaller dimensions should be lower than $10 \%$. The decision to include obstacles or not will depend on

403 the exact order of magnitude of the other sources of uncertainty, the number, size and location of the

404 obstacles and finally the availability of obstacles data.

\section{Notation}

$406 \quad b \quad=\quad$ channel width $(\mathrm{m})$

$407 C=$ weir crest height $(\mathrm{m})$

$408 f=$ friction factor $(-)$

$409 \mathrm{~F}=$ Froude number (-)

$410 \mathrm{~g}=\quad$ constant of gravity $\left(\mathrm{m} \mathrm{s}^{-2}\right)$

$411 h=\quad$ water depth $(\mathrm{m})$

$412 K=$ eddy viscosity coefficient $\left(\mathrm{m}^{2} \mathrm{~s}^{-1}\right)$

$413 K_{s}=$ Strickler coefficient $\left(\mathrm{m}^{1 / 3} \mathrm{~s}^{-1}\right)$

$414 k_{\mathrm{s}}=$ roughness height $(\mathrm{m})$

$415 m=$ mesh resolution $(\mathrm{cm})$

$416 n=$ Manning roughness coefficient $\left(\mathrm{s} \mathrm{m}^{-1 / 3}\right)$

$417 Q=$ discharge $\left(\mathrm{m}^{3} \mathrm{~s}^{-1}\right)$

$418 Q^{*}=$ discharge distribution error $(\%)$

$419 \mathrm{R}=$ Reynolds number $(-)$

$420 \quad R_{h}=$ hydraulic radius (m)

$421 \quad R_{q} \quad=\quad$ discharge distribution (-)

$422 t=$ time $(\mathrm{s})$

$423 u, v=$ velocity components $\left(\mathrm{m} \mathrm{s}^{-1}\right)$ 


$\begin{array}{llll}424 & Z_{b} & = & \text { bottom elevation }(\mathrm{m}) \\ 425 & & = & \text { elevation }(\mathrm{m}) \\ 426 & \delta & = & \text { average } \\ 427 & v & = & \text { kinematic viscosity }\left(\mathrm{m}^{2} \mathrm{~s}^{-1}\right) \\ 428 & \sigma & = & \text { root mean square deviation } \\ 429 \Delta R_{q} & = & \text { discharge modification }(\%)\end{array}$

430

431 Subscripts

$\begin{array}{llll}432 u & = & \text { upstream channel } \\ 433 & d & = & \text { downstream channel } \\ 434 & b & = & \text { branch channel } \\ 435 & 0 & = & \text { without obstacle } \\ 436 & 1-7 & = & \text { obstacle number } \\ 437 & 10 & = & \text { with sidewalks } \\ 438 & \text { SIM } & = & \text { simulated } \\ 439 & \text { MES } & = & \text { measured }\end{array}$

\section{References}

442 Bazin, P.H. (2013). Flows during floods in urban areas: Influence of the detailed topography and 443 exchanges with the sewer system ( $\mathrm{PhD}$ thesis). University Claude Bernard - Lyon I, Lyon. 444 Bravo, H. R., \& Holly, F. M. (1996). Turbulence model for depth-averaged flows in navigation 445 installations. Journal of Hydraulic Engineering, ASCE, 122(12), 718-727.

446 EEA (2010). Mapping the impacts of natural hazards and technological accidents in Europe An 447 overview of the last decade. Copenhagen, Denmark, European Environment Agency (EEA), 146. 448 El Kadi Abderrezzak, K., Paquier, A., \& Mignot, E. (2009). Modelling flash flood propagation in 449 urban areas using a two-dimensional numerical model. Natural Hazards, 50(3), 433-460.

450 El Kadi Abderrezzak, K., Lewicki, L., Paquier, A., Riviere, N., \& Travin, G. (2011). Division of 451 critical flow at three-branch open-channel intersection. Journal of Hydraulic Research, 49(2), 452 231-238.

453 Ghostine, R., Mignot, E., Abdallah, M., Lawniczak, F., Vazquez, J., Mose, R., \& Gregoire, C. (2010). 454 Discontinuous Galerkin Finite-Element Method for Simulation of Flood in Crossroads. Journal 455 of Hydraulic Engineering, ASCE, 136(8), 474-482.

456 Jiang, C., Yang, C., \& Liang, D. (2009). Computation of shallow wakes with the fractional step finite 457 element method. Journal of Hydraulic Research, 47(1), 127-136. 
Khan, A. A., Cadavid, R., \& Wang, S. S. Y. (2000). Simulation of channel confluence and bifurcation using the CCHE2D model. Proceedings of the Institution of Civil Engineers-Water Maritime and Energy, 142(2), 97-102.

Leandro, J., Chen, A. S., Djordjevic, S., \& Savic, D. A. (2009). Comparison of 1D/1D and 1D/2D Coupled (Sewer/Surface) Hydraulic Models for Urban Flood Simulation. Journal of Hydraulic Engineering, ASCE, 135(6), 495-504.

Lloyd, P. M., \& Stansby; P. K. (1997). Shallow-water flow around model conical islands of small side slope .1. Surface piercing. Journal of Hydraulic Engineering, ASCE, 123(12), 1057-1067.

Mignot, E., Paquier, A., \& Haider, S. (2006). Modeling floods in a dense urban area using 2D shallow water equations. Journal of Hydrology, 327(1-2), 186-199.

Mignot, E., Paquier, A., \& Ishigaki, T. (2006). Comparison of numerical and experimental simulations of a flood in a dense urban area. Water Science and Technology, 54(6-7), 65-73.

Mignot, E., Paquier, A. \& Riviere, N. (2008). Experimental and numerical modeling of symmetrical four-branch supercritical cross junction flow. Journal of Hydraulic Research, 46(6), 723-738.

Mignot, E., Riviere, N., Perkins, R., \& Paquier, A. (2008). Flow patterns in a four-branch junction with supercritical flow. Journal of Hydraulic Engineering, ASCE, 134(6), 701-713.

Mignot, E., Zeng, C., Dominguez, G., Li, C.-W., Rivière, N., \& Bazin, P.-H. (2013). Impact of topographic obstacles on the discharge distribution in open-channel bifurcations. Journal of Hydrology, 494(28), 10-19.

Mignot, E., Doppler, D., Riviere, N., Vinkovic, I., Gence, J.N., \& Simoens, S. (2014). Analysis of flow separation using a local frame-axis: application to the open-channel bifurcation. Journal of Hydraulic Engineering, ASCE, 140 (3), 280-290

Papanicolaou, A. N., Elhakeem, M., \& Wardman, B. (2011). Calibration and Verification of a 2D Hydrodynamic Model for Simulating Flow around Emergent Bendway Weir Structures. Journal of Hydraulic Engineering, ASCE 137(1), 75-89.

Paquier, A.,\& Bazin, P.H. (2014). Estimating uncertainties for urban floods modelling. Houille Blanche-Revue Internationale de l'eau, 6, 13-18.

Shettar, A. S., \& Murthy, K. K. (1996). A numerical study of division of flow in open channels. Journal of Hydraulic Research, 34(5), 651-675.

Soares-Frazão, S., \& Zech, Y. (2008). Dam-break flow through an idealised city. Journal of Hydraulic Research, 46(5), 648-658.

Stansby, P. K. (2006). Limitations of depth-averaged modeling for shallow wakes. Journal of Hydraulic Engineering, ASCE, 132(7), 737-740.

Van Emelen, S., Soares-Frazao, S., Riahi-Nezhad, C. K., Chaudhry, M. H., Imran, J., \& Zech, Y. (2012). Simulations of the New Orleans 17th Street Canal breach flood. Journal of Hydraulic Research, 50(1), 70-81. 
494 Yen, B. C. (2002). Open Channel Flow Resistance. Journal of Hydraulic Engineering, ASCE, 128(1), 495 20-39.

496 Yulistiyanto, B., Zech, Y. \& Graf, W. H. (1998). Flow around a cylinder: Shallow-water modeling 497 with diffusion-dispersion. Journal of Hydraulic Engineering, ASCE, 124(4), 419-429 498 
Table 1: Main parameters of the 14 flow configurations without obstacles.

\begin{tabular}{|c|c|c|c|c|c|}
\hline Series & Flow & $\begin{array}{l}Q_{u 0} \\
\mathrm{Ls}^{-1}\end{array}$ & $\begin{array}{l}\mathrm{F}_{\mathrm{u} 0} \\
-\end{array}$ & $\begin{array}{l}R_{q 0} \\
-\end{array}$ & $\begin{array}{l}h_{u 0} / b \\
-\end{array}$ \\
\hline \multirow{6}{*}{ S1 } & 1 & 6.01 & 0.79 & 0.39 & 0.13 \\
\hline & 2 & 4.99 & 0.60 & 0.39 & 0.14 \\
\hline & 3 & 4.01 & 0.45 & 0.39 & 0.15 \\
\hline & 4 & 3.00 & 0.33 & 0.39 & 0.15 \\
\hline & 5 & 2.51 & 0.28 & 0.40 & 0.15 \\
\hline & 6 & 2.00 & 0.23 & 0.38 & 0.14 \\
\hline \multirow{5}{*}{ S2 } & 7 & 4.00 & 0.44 & 0.23 & 0.15 \\
\hline & 3 & 4.01 & 0.45 & 0.39 & 0.15 \\
\hline & 8 & 4.00 & 0.45 & 0.51 & 0.15 \\
\hline & 9 & 4.00 & 0.44 & 0.65 & 0.15 \\
\hline & 10 & 3.99 & 0.45 & 0.80 & 0.15 \\
\hline \multirow{5}{*}{ S3 } & 11 & 1.66 & 0.44 & 0.40 & 0.08 \\
\hline & 12 & 2.77 & 0.45 & 0.38 & 0.12 \\
\hline & 3 & 4.01 & 0.45 & 0.39 & 0.15 \\
\hline & 13 & 5.38 & 0.45 & 0.39 & 0.18 \\
\hline & 14 & 7.00 & 0.45 & 0.39 & 0.22 \\
\hline
\end{tabular}

500

501 Table 2: Numerical parameters for the 7 runs and simulation quality indicators without obstacle.

\begin{tabular}{lllll} 
Run & $m$ & $K$ & $\delta\left(Q_{b 0}^{*}\right)$ & $\sigma\left(Q_{b 0}^{*}\right)$ \\
& $\mathrm{cm}$ & $\mathrm{m}^{2} \mathrm{~s}^{-1}$ & $\%$ & $\%$ \\
\hline A & $3.5-5$ & 0 & -1.88 & 2.50 \\
B & $3.5-5$ & $5.0 \times 10^{-4}$ & -1.73 & 2.34 \\
$\mathrm{C}$ & $3.5-5$ & $1.0 \times 10^{-3}$ & -1.71 & 2.36 \\
\hline E1 & 0.5 & 0 & -0.98 & 1.75
\end{tabular}


502

$\begin{array}{lllll}\text { E2 } & 0.5 & 2.0 \times 10^{-4} & -1.19 & 2.14 \\ \text { D } & 0.5 & 5.0 \times 10^{-4} & -0.99 & 1.99 \\ \text { E3 } & 0.5 & 1.0 \times 10^{-3} & -1.11 & 1.97\end{array}$

503 Table 3: Numerical parameters for the 4 runs and simulation quality indicators with obstacle.

\begin{tabular}{lllllll} 
& $m$ & $K$ & $\delta\left(\Delta R_{q 1-7}\right)$ & $\sigma\left(\Delta R_{q 1-7}\right)$ & $\delta\left(Q_{b 1-7}^{*}\right)$ & $\sigma\left(Q_{b 1-7}^{*}\right)$ \\
& $\mathrm{cm}$ & $\mathrm{m}^{2} \mathrm{~s}^{-1}$ & $\%$ & $\%$ & $\%$ & $\%$ \\
\hline A & $3.5-5$ & 0 & -0.37 & 1.13 & -2.80 & 3.79 \\
B & $3.5-5$ & $5.0 \times 10^{-4}$ & -0.01 & 0.86 & -1.70 & 2.71 \\
$\mathrm{C}$ & $3.5-5$ & $1.0 \times 10^{-3}$ & 0.17 & 0.85 & -1.21 & 2.67 \\
$\mathrm{D}$ & 0.5 & $5.0 \times 10^{-4}$ & -0.08 & 0.71 & -1.27 & 2.50 \\
\hline
\end{tabular}

504

505

506 Table 4: Numerical parameters and indicators on the discharge distribution evolution for the 507 simulations of flows with sidewalks

\begin{tabular}{lllllll} 
& & $K$ & $\delta\left(\Delta R_{q 10}\right)$ & $\sigma\left(\Delta R_{q 10}\right)$ & $\delta\left(Q_{b 10}^{*}\right)$ & $\sigma\left(Q_{b 10}^{*}\right)$ \\
& & $\mathrm{m}^{2} \mathrm{~s}^{-1}$ & $\%$ & $\%$ & $\%$ & $\%$ \\
\hline 1 & Ref & $5.0 \times 10^{-4}$ & -0.21 & 2.08 & -2.24 & 5.72 \\
2 & Ref & $1.0 \times 10^{-3}$ & 0.01 & 1.88 & -1.14 & 5.28 \\
3 & Avg & $5.0 \times 10^{-4}$ & -0.48 & 2.05 & -2.90 & 5.58 \\
4 & $A v g$ & $1.0 \times 10^{-3}$ & -0.37 & 1.99 & -2.30 & 5.32
\end{tabular}

508 
511 Figure 1: Obstacles (a) and sidewalks (b) configurations

513 Figure 2: a: Measured velocities at elevation $z=3 \mathrm{~cm}$ (Exp) and simulated depth averaged velocities

514 (runs A, B, C, D) around the crossroad for flow 6 without obstacle. For the fine mesh run, only a

515 selection of the computed velocities is shown. b: Comparison of the measured and simulated (using

516 runs B and D) of the streamwise ( $u$ ) and transverse ( $v$ ) mean velocity components along the $x=0.15 \mathrm{~m}$

517 profile.

519 Figure 3: Extent of the recirculation zones measured at $z=3 \mathrm{~cm}$ (Exp) and computed (depth averaged)

520 for runs $\mathrm{A}(K=0)$ and $\mathrm{C}\left(K=10^{-3} \mathrm{~m}^{2} \mathrm{~s}^{-1}\right)$, for flow 6 without obstacles.

522 Figure 4: Comparison of simulated (SIM) and measured (MES) evolutions of discharge distribution for 523 each obstacle for runs B (coarse mesh) and D (fine mesh). Result of the linear regression is indicated 524 with the grey line, along with its slopes.

525

526 Figure 5: Measured ( $\mathbf{a})$ and simulated (runs B with coarse mesh $(\Delta)$ and D with fine mesh (o))

527 evolutions of the discharge distribution for each obstacle configuration (7 lines) and each flow

528 grouped according to the 3 series (3 columns).

530 Figure 6: a: Measured velocities at elevation $z=3 \mathrm{~cm}$ (Exp) and simulated depth averaged velocities

531 (runs A, B, C and D) around the crossroad for flow 6 with obstacle 2. b: Comparison of the measured

532 and simulated (using runs B and D) of the streamwise $(u)$ and transverse $(v)$ mean velocity

533 components along the $x=0.15 \mathrm{~m}$ profile; note that runs $\mathrm{A}$ and $\mathrm{C}$ exhibit quite similar velocity profiles as 534 run B and were thus omitted for sake of simplicity.

536 Figure 7: Top view of the mesh (left) and cross section (right, shown along the dotted line on the left

537 plot) with the channel bottom elevation used in models Ref and Avg for flows with sidewalks 538 simulations.

540 Figure 8: Measured at $\mathrm{z}=3 \mathrm{~cm}$ (Exp) and simulated (depth averaged, with runs 1 and 2) flow velocity 541 magnitude for flow 6 with sidewalks: around the junction (a), 2D velocity components along the $542 x=0.15 \mathrm{~m}$ profile (b) and extent of the recirculation zones (c). 
544 Figure 9: Measured (Exp) and simulated (runs 1, 2, 3 and 4) evolution of the flow discharge

545 distribution for the 14 flows with sidewalks

546

547 Figure 10: Computed (with run 1) water levels around the junction for flows 1, 2 and 3 without

548 sidewalks (top) and with sidewalks (bottom). Supercritical flow areas ( $\mathrm{F}>1$ ) are shown as hashed. 549 